\title{
Effects of pressure decay on Non-Methane Volatile Organic Compounds (NMVOC) species distribution in domestic aerosol sprays with LPG propellants.
}

\author{
Muhammad Kabir Abba*1, Zainab Naeem ${ }^{1}$, Amir Nourian ${ }^{1}$, Ghasem Ghavami Nasr ${ }^{1}{ }^{1}$ \\ ${ }^{1}$ Spray Research Group (SRG), School of Science Engineering and Environment (SEE), \\ University of Salford, Manchester, UK. \\ ${ }^{*}$ Corresponding author email: $\underline{\text { m.k.abba1@salford.ac.uk }}$
}

\begin{abstract}
Conventional aerosols sprays contain different Volatile Organic Compounds (VOC). These organic compounds have different properties and can have detrimental impact on air quality. This study investigated the spray performance and variation of the types of Non-Methane VOCs (NMVOCs) expelled over the life of pressurised aerosol spray can. Three types of aerosol sprays - hair sprays, deodorants, and antiperspirants were selected from the solventbased cosmetic products. Mass Ratio (MR) of solvent (ethanol) to propellant (LPG) for all the products was analysed at pressure decay sequences of $4,3,2$, and 1.5 bar. It was found that the MR in hair sprays expelled was significantly higher than that of antiperspirants and body sprays by $53 \%$ and $54 \%$ respectively at 4 bar. As the pressure depleted, however, the antiperspirant and body sprays' MR decreased while that of the hair spray increased. At the lower pressures (2 bar), the hair spray had the highest MR and antiperspirant had the lowest. This finding is important in evaluating the performance of the delivery pathways of aerosol spray products and will provide insight into the effective design of atomisers with alternative "green" propellants like compressed air replacing LPG as the primary propellant in spray products.
\end{abstract}

\section{Keywords}

NMVOC, Solvent, Species Distribution, Pressure Decay, Propellant

\section{Introduction}

Aerosol spray is a dispensing system which creates mist of liquid or fine solids. This is achieved through the pressurisation of the active ingredient in the containing vessel using a gas [1]. The pressurising gas is called the propellant. About $80 \%$ of aerosol dispensers use Liquefied Petroleum Gas (LPG) as propellant [2]. These aerosol spray cans contain different components which are Non-Methane Volatile Organic Compounds (NMVOC) which have recently been shown to have impact on air quality, health, and environment [3]. A typical pressurised aerosol spray can is shown in Figure 1. 


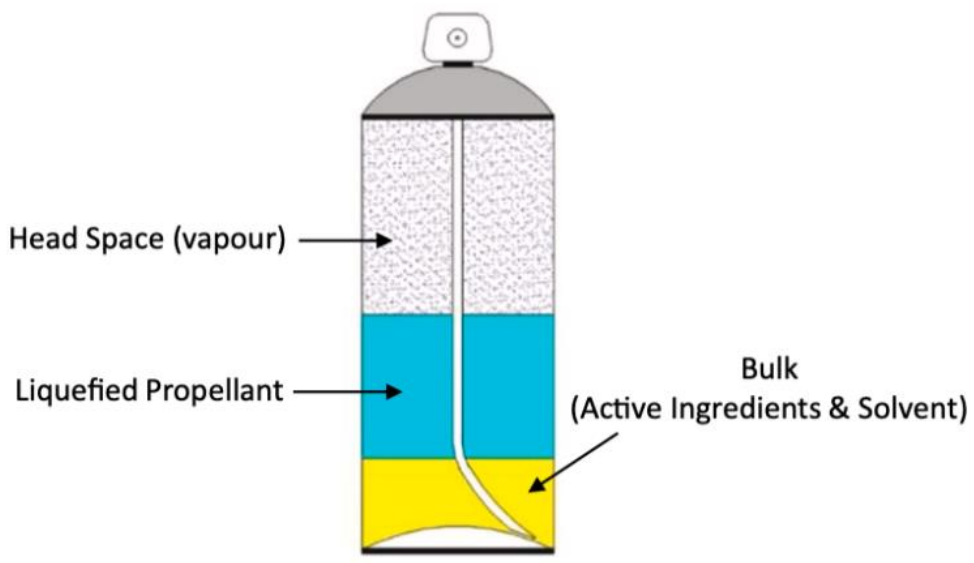

Figure 1. Schematics of a typical pressurised aerosol spray can [4]

Aerosol sprays are utilised in a wide variety of applications and the major reason is the ease with which it is operated and its low cost as there is no need for any additional equipment [5]. Domestic aerosol sprays make up about $70 \%$ of the global annual production [4] which includes household and personal care products. Aerosol sprays extend into other areas of application such as lubrications and paints which contain several other harmful NMVOCs.

The evolution and adoption of current LPG propellant stem from the ban of Chlorofluorocarbons (CFC) following the Montreal Protocol of 1987. However, a newer call for decarbonisation is on the rise and industry giants are taking the necessary steps towards the revolutionary Net-Zero drive by 2050 . This includes the aerosol industry's replacement of LPG propellant with environmentally friendly, "clean", alternatives such as Nitrogen or compressed air. This changeover, however, will not be as straight forward as the switch from CFCs to LPG given that CFC and LPG have similar thermodynamic properties. The major advantage is the "flashing" property of CFC and LPG which is desirable especially in personal care spray products category. For "clean" inert gas as propellant in pressurised aerosols, that "flashing" property is absent and thus higher energy is required for atomisation of the liquid. Following this, the spray distribution and dispensation will certainly not be these same as that of the LPG. Therefore, research is ongoing to improve the dynamics of the "clean" compressed air in pressurised aerosols and make the switch from LPG without compromising efficiency.

Several works have been carried out to evaluate the spray performance of the pressurised aerosol sprays. Nourian et al. [6][7][8] compared the spray performance between compressed air and LPG propellants in pressurised aerosols cans. They presented novel valve designs that will improve the efficiency of compressed air propelled aerosol products in terms of fluid type and spray duration. The pressure consistency of LPG propellant (see Figure 2) was showcased which was associated with droplet size consistency during application unlike the variation in droplet sizes as in the case of compressed air. Furthermore, the identification, distribution, and impact of the NMVOCs contained in conventional aerosol sprays is essential and will highlight the dominance of the most active component in the propellant. It proceeds to enable the detection of the species that would have profound impact on the atomisation of the bulk products based on viscosity, density, and any other property that affects atomisation. Quantification, thus, will provide an overview of the magnitude of potentially hazardous emissions from the use of pressurised aerosols containing NMVOCs. It has been reported [4] that personal care products category, specifically body sprays, contains $93.7 \mathrm{wt} \%$ NMVOCs with more than $60 \mathrm{wt} \%$ coming from the propellants alone (LPG). Therefore, the replacement 
of the LPG propellant with clean alternative such as nitrogen or compressed air will undoubtedly reduce the emissions from household products by a staggering $60 \%$.

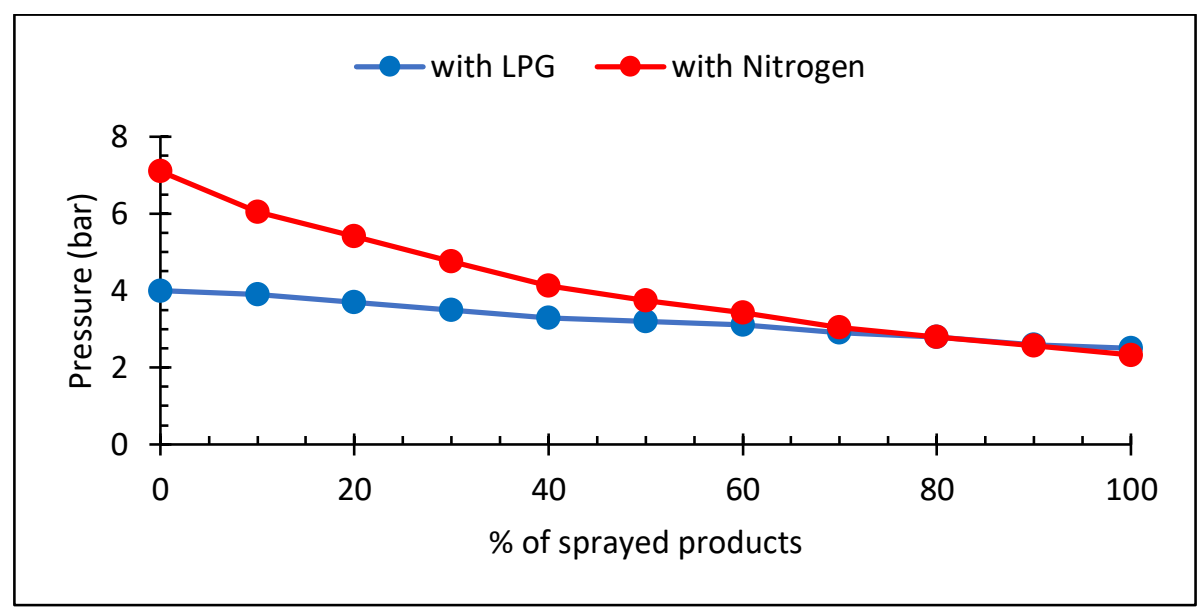

Figure 2. Pressure depletion comparison for a typical aerosol spray with LPG and Nitrogen propellants [7]

Focus has always been on the droplet sizes with respect to pressure decline during the life of the pressurised can. Another important aspect of spray characterisation is the type of specie expelled with respect to other parameters such as pressure decline. This overarching question can be asked; Does pressure affect the species distribution upon application? Another important approach to this question is the relationship between the propellant and the bulk of the products in the pressurised can which contains the solvent. It is a well-known fact that the consistency of spray in LPG propelled aerosol products is unmatched (as seen in Figure 2 and thus the product distribution is even throughout the duration of the spray. To this end, the interplay between the pressure and species distribution will be investigated. Studies of this type are very important in the drive to replace LPG with clean alternatives. They will provide an understanding of the mechanics involved during to the application and help improve the current technology for efficient deployment of the technology in order to achieve the Net-zero future.

\section{Material and Methods}

For this study, off the shelve personal care (cosmetics) spray products were utilised. The female targeted products consisting of body sprays, antiperspirants, and hair sprays were selected because of the adoption of consistency and are more widely used than the male targeted. To highlight the consistency of the methodology and experimental setup, three (3) cans of each type of cosmetic spray can were employed. Table 1 shows the selection of the female targeted cosmetic spray products.

Table 1 - Selected female targeted aerosol sprays.

\begin{tabular}{c|ccc}
\hline $\begin{array}{c}\text { Cosmetic } \\
\text { products }\end{array}$ & $\begin{array}{c}\text { Sizes } \\
(\mathrm{ml})\end{array}$ & $\begin{array}{c}\text { Gross } \\
\text { Mass }(\mathrm{g})\end{array}$ & $\begin{array}{c}\text { No. of } \\
\text { Cans tested }\end{array}$ \\
\hline Body Spray & 75 & 67.5 & 3 \\
Antiperspirant & 150 & 124.3 & 3 \\
Hair Spray & 100 & 95.3 & 3
\end{tabular}


The experimental setup consists of a graduated glass measuring cylinder which was used as the sampling chamber, two high accuracy mass balances for measuring solvent and product masses, a Gas Chromatograph (6890N) and Mass Spectrometer (5873N) from Agilent UK for analysis, and a computer for pre- and post-processing. Details of the method and procedures are detailed elsewhere in our previous work [4]. The only distinguishing feature between this work and our previous work is the intermittent pressure measurement. Here, the pressures in the can were measured using a GE pressure digital transducer $(0.01 \%)$. Before each spray, the can pressure was measured by placing the transducer on the can and depressed to get a reading, taking care not to spill the contents. The pressure was then recorded and the aerosol was then sprayed into the sampling chamber for the analysis using a developed method in the GCMS. As the graduated sampling chamber is airtight, the sprayed aerosol pressurises the chamber and elevates the pressure to about 2-3 atm (as the spray duration into the chamber remains 5 seconds). At this pressure, condensation of the effluent from the aerosol occurs. Thus, the mass and volume of the liquid components can then be measured and recorded, against the GCMS analysed vapour components. This step was carried out until the aerosol can is emptied into the chamber. The setup is shown in Figure 3.

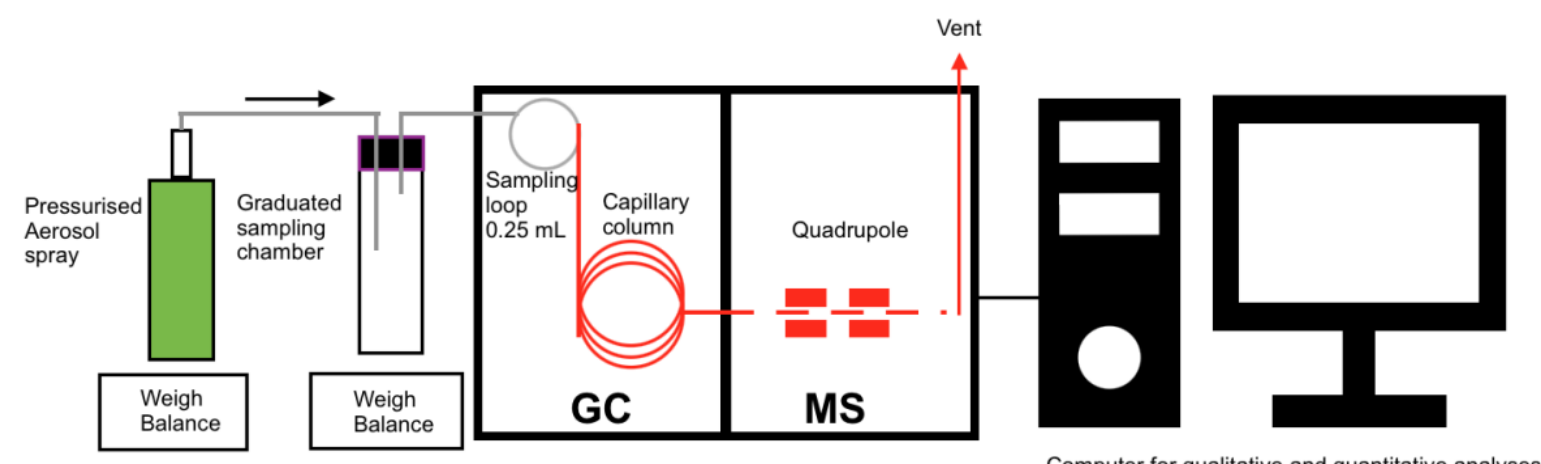

Figure 3. Schematics of the experimental set up

\section{Results and Discussion}

Evaluation of Solvent to Propellant Mass Ratio (MR)

The MR shows the interplay between the solvent and propellant masses as they are expelled from the pressurised spray can. This is highly dependent on the pressure of atomisation of the solvent by the propellant which dictates the droplet sizes and other spray characteristics. Atomisation is the process of breaking up bulk liquids into droplets. The mass ratio is evaluated using Equation (1).

$$
M R=\frac{\text { Mass of Solvent }(g)}{\text { Mass of Propellant }(g)}=\frac{M_{S}}{M_{P}}
$$

At each stage of the pressure decline, the flowrates, and the masses of solvent and propellant dispelled were measured and used to evaluate the MR for all the tested aerosol products. Figure 4 shows the mass flowrates of each pressurised can as the pressure declines. There was a degree of consistency in the mass flowrates especially for the body spray and the antiperspirants. This can be attributed to the fill ratio of the products which is the amount of the bulk to that of propellant during filling process. The higher the fill ratio the lower the 
consistency of the flow rate as it will require additional energy to convey the usually heavier constituents of the can by the lower density propellant. Another factor that may affect the consistency of the spray is the viscosity of the bulk component [9]. Conversely, the hair spray contains polymers and holding agents which may increase the viscosity of the bulk. This may explain the inconsistency of the flowrate as the pressure declined upon application in Figure 4 for Hair Spray.

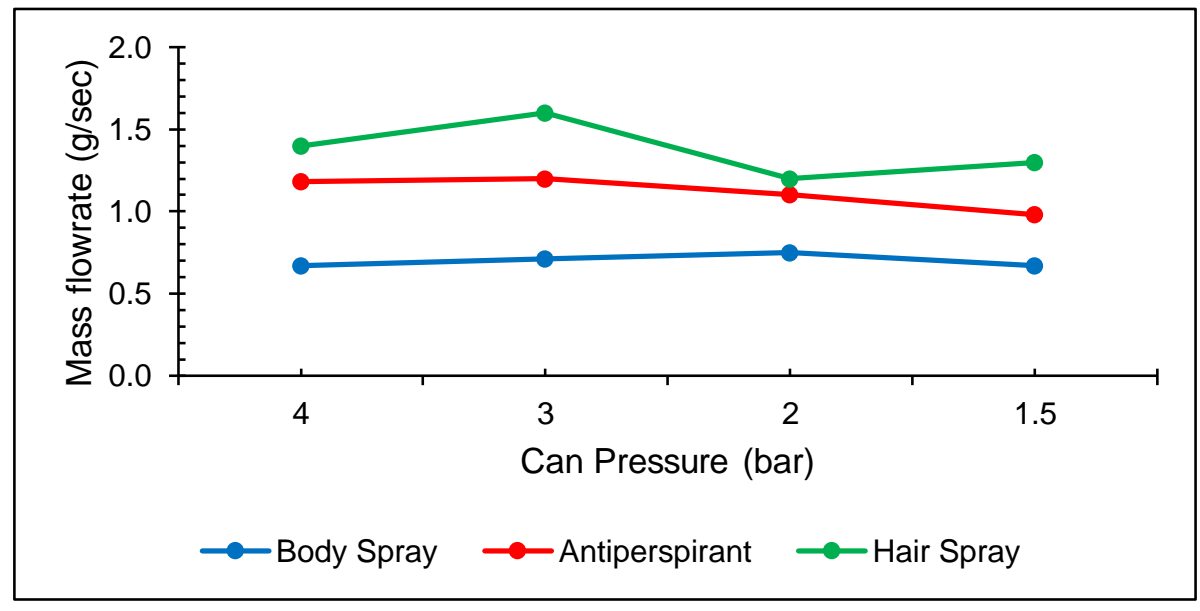

Figure 4. Mass flowrate vs pressure depletion for all the test cans

Figure 5 shows the MR for all the cans tested. The MR in body spray showed that at the onset of the spray (4 bar), the amount of solvent is lower than the propellant. As the pressure declined, the solvent amount in the spray also declined till at about 2 bar pressure. The solvent amount then began to gradually increase until the can equilibrated with the atmospheric pressure. Once that happened, the dispensing system stopped as there was no pressure difference between the pressurised can and ambient pressure. Similar trend was observed in the antiperspirant experiment albeit having higher magnitude of flowrate and significantly lower MR compared to the body spray counterpart. This can still be credited to the lower masses of solvent compared to the propellant as highlighted by Nourian et al [4] and variation in the fill ratio of the antiperspirant and as such will have higher mobility.

Interestingly, the MR of the hair spray is exhibiting an outlier trend in that it deviated expansively from those of the body spray and antiperspirant. The MR increased as the pressure declined and was highest at 2 bar pressure before rapidly declining at 1.5 bar. This means the most bulk product was dispensed in the middle of the life of the aerosol spray. In Figure 4 and 5, at 2 bar pressure, the mass flowrate was lowest, and the MR was highest at this point for hair spray. This can be explained by the fact that the almost 2 times the mass of the propellant was expelled from the can as lower pressures from the opening 4 bar pressure. This was characterised by streaking of the product as it approached empty can mark. It alludes that the energy required to expel more product has waned significantly and the atomisation did not produce fine sprays upon depressing the actuator. Another observation is that, throughout the experiment of the hair spray, the MR was above $1 \mathrm{MR}$, signifying that more solvent is expelled compared to the propellant. This design might be because of the application of the product given it needs to cover larger surface area. 


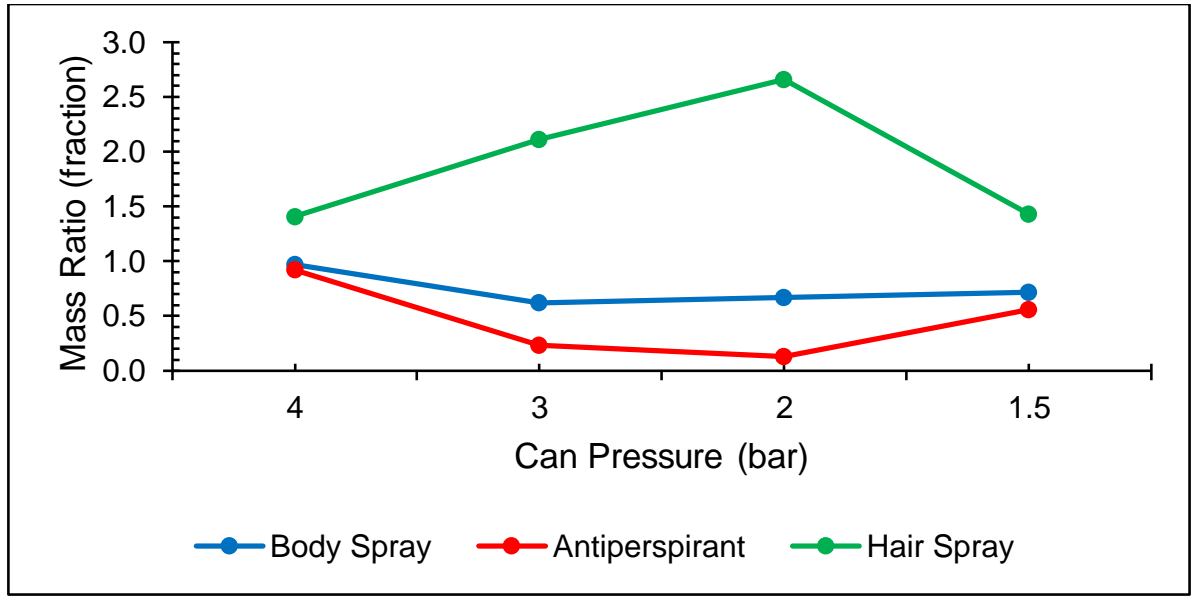

Figure 5. Relationship between Mass Ratio (MR) and the pressure depletion for tested cans

This approach for spray performance evaluation will help determine the NMVOC species distribution during application of the products. Thus, the analysis of the atomised products to show the distribution of the species as pressure declines with use will provide additional layer to the performance evaluation.

\section{Species distribution evaluation with respect to pressure decline}

Using the developed method in the GCMS, a careful analytical analysis of the constituents of the spray was performed. This enabled the identification and quantification of the NMVOCs in the atomised products of the aerosols. Figure 6 shows the distribution of the body spray.

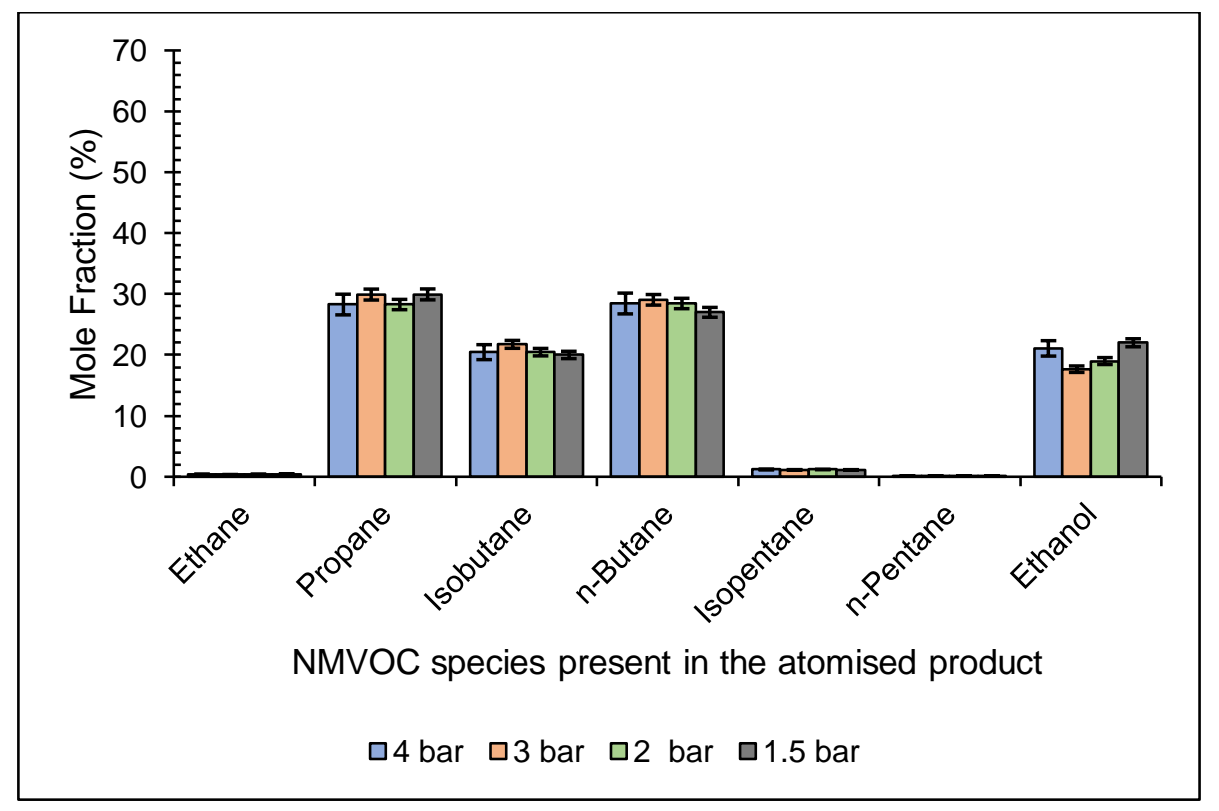

Figure 6. NMVOC species distribution as a function of pressure depletion for body spray

The distribution shows that as pressure decreases, the heavier gaseous components decrease as seen in the $n$-butane stack. Ethanol was identified as the primary solvent in the body spray and as expected, the trend followed the same trend as the MR for body spray where the mass of the ethanol was significant at the onset of the spray and then decreased as pressure declined and then increases near the empty mark of the pressurised can. In Figure 
7, there was noticeably no ethanol present in the product distribution. The propellant served as both solvent and propellant in the design as it contains drying agents which takes advantage of the flash vaporisation of the LPG for effectiveness. Propane was predominant and appeared to increase with decrease in pressure. Other components, like butane and its isomer, isobutane, also followed the same trends. Figure 8 shows the hair spray distribution which is similar to the body spray. Only difference is higher ethanol content than body spray and can be explained by reasons given in the MR section discussion.

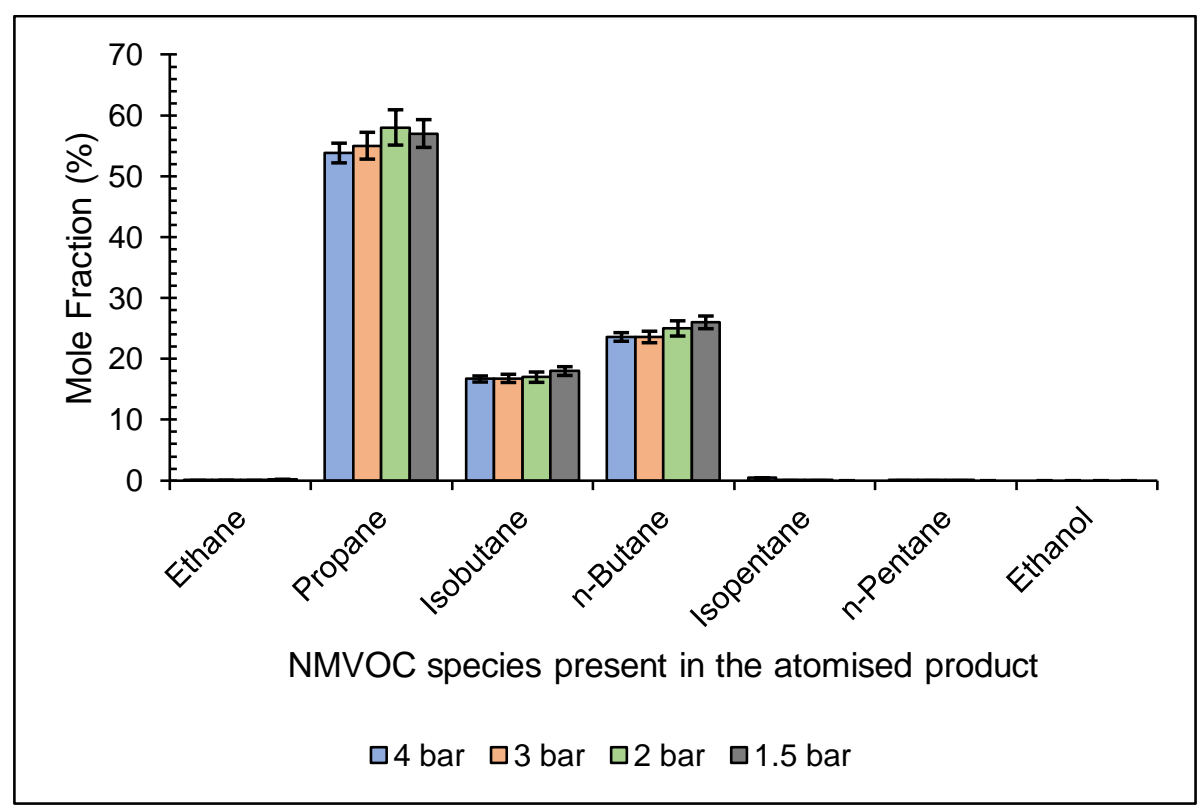

Figure 7. NMVOC species distribution as a function of pressure depletion for antiperspirant

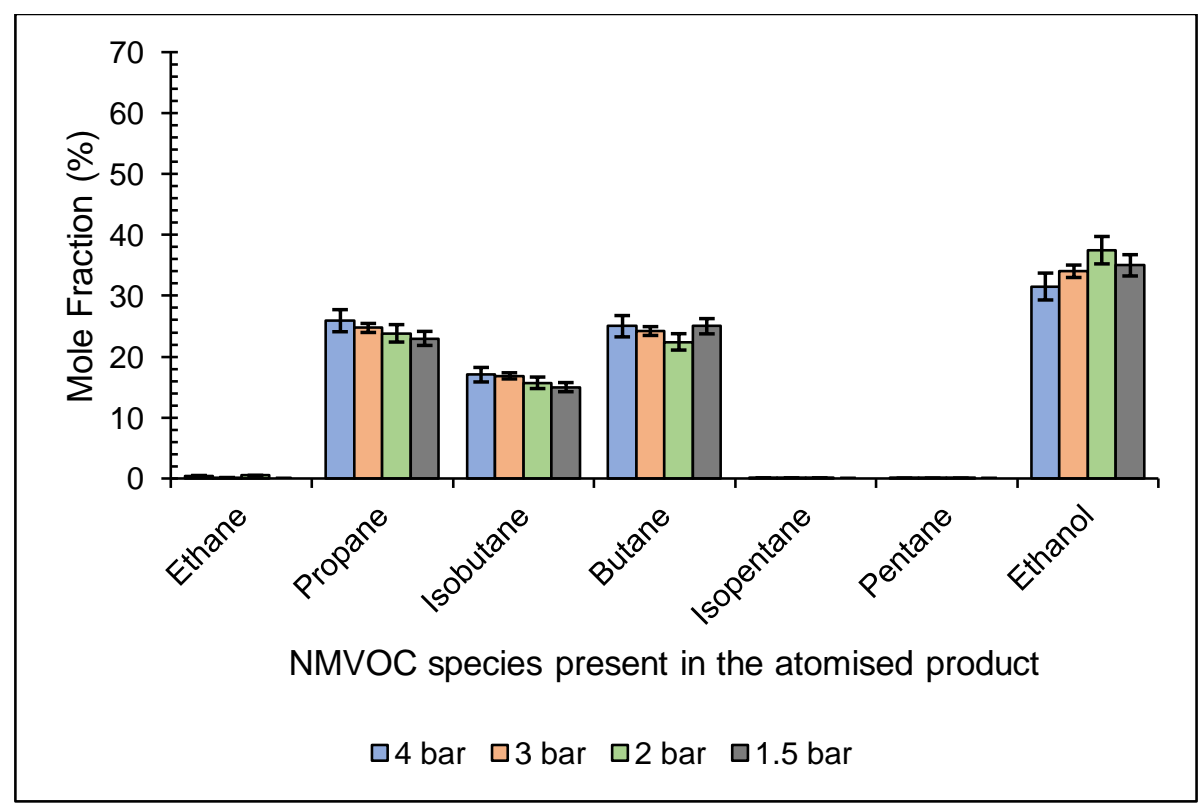

Figure 8. NMVOC species distribution as a function of pressure depletion for hair spray

\section{Conclusions}

A demonstration of the spray performance of conventional pressurised spray product was carried out using the Mass Ratio (MR) approach. Hair spray showed higher MR than 
antiperspirant and body sprays with antiperspirant exhibiting the lowest MR. The NMVOC species distribution was also described. Antiperspirants demonstrated that as pressure decreases, the mole fractions of the gaseous components increase when LPG propellants are used.

This study will help identify adjustments to be made and noted when transitioning from LPG propellants to compressed air or nitrogen. The effectiveness of the spray product depends on the spray performance in product distribution. Care must thus be taken in the design of the dispensing system including the atomiser and valves. With efforts aimed at the decarbonisation of the aerosol industry, adopting "green" alternatives to conventional constituents in aerosol sprays is the reliable avenue to achieving a net "zero" future without compromising the effectiveness and consumer satisfaction.

\section{Acknowledgments}

This work was financially supported by the School of Science, Engineering and Environment (SEE) at University of Salford, Manchester, UK.

\section{Nomenclature \\ $M_{s} \quad$ mass of solvent $[\mathrm{g}]$ \\ $M_{P} \quad$ mass of propellant [g]}

\section{References}

[1] A. J. Yule, "The household aerosol: present and future of the world's largest market for spray technology," in Proc. 9th International Conference on Liquid Atomization and Spray Systems (ICLASS 2003), Invited Lectures, 2003, no. July 2003.

[2] P. Floyd, B. Georgalas, S. Marshall, C. Elding, and Risk and Policy Analysts (RPA), "Impact Assessment Study on the Adaptation to Technical Progress of the Aerosol Dispensers Directive (Final Report)," 2014.

[3] The Royal College of Paediatrics and Child Health $(\mathrm{RCPCH})$ et al., "The inside story: Health effects of indoor air quality on children and young people," 2020.

[4] A. Nourian, M. K. Abba, and G. G. Nasr, "Measurements and analysis of non-methane VOC (NMVOC) emissions from major domestic aerosol sprays at 'source,"' Environ. Int., vol. 146, p. 106152, 2021.

[5] H. Kim, J. Kim, and S. Park, "Atomization characteristics of aerosol spray from hair spray vessel with various design parameters," J. Aerosol Sci., vol. 133, no. April, pp. 24-36, 2019.

[6] A. Nourian, G. Nasr, D. Pillai, and M. Waters, "Compressed gas domestic aerosol valve design using high viscous product," Int. J. Multiphys., vol. 8, no. 4, pp. 437-460, 2014.

[7] A. Nourian, G. G. Nasr, A. J. Yule, G. Hawthorne, and T. Goldberg, "Novel metered aerosol valve," Proc. Inst. Mech. Eng. Part C J. Mech. Eng. Sci., vol. 230, no. 10, pp. 1557-1568, 2016.

[8] A. Nourian, G. G. Nasr, A. J. Yule, T. Goldberg, and G. Tulloch, "Next generation of consumer aerosol valve design using inert gases," Proc. Inst. Mech. Eng. Part C J. Mech. Eng. Sci., vol. 229, no. 16, pp. 2952-2976, 2014.

[9] I. Graco, "Atomization Concept and Theory," 1995. 